Negat i ve Absor pti on near the El ectron Cycl ot r on Har moni cs i n Weakl y I oni zed Gases

\begin{tabular}{|l|l|}
\hline $\begin{array}{l}\text { j our nal or } \\
\text { publ i cat i on } \mathrm{t} \text { i t l e }\end{array}$ & 福井大学工学部研究報告 \\
\hline Vol une & 17 \\
\hline number & 2 \\
\hline page $r$ ange & $201-211$ \\
\hline year & $1969-09$ \\
\hline URL & ht t p: //hdl . handl e. net /10098/4830 \\
\hline
\end{tabular}




\title{
Negative Absorption near the Electron Cyclotron \\ Harmonics in Weakly lonized Gases
}

\author{
Toshitaka Idehara*, Katsuji Nakaya* \\ and Yoshio IshidA*
}

(Received Apr. 15, 1969)

\begin{abstract}
Negative absorption of radio frequency wave was observed near the electron cyclotron harmonics $\left(f=n f_{c}\right)$ in weakly ionized plasma, and the voltage gains near $f=f_{c}$ and $f=2 f_{c}$ were about $7 d B$ and about $4 d B$, respectively. The emissions near $f=n f_{c}$ ascribed to this phenomenon were observed up to $n=4$ in Xe-plasma, up to $n=2$ in $\mathrm{Kr}$-plasma and only at $n=1$ in Ar-plasma.

Their powers were stronger by several tens $d B$ than that of thermal noise. Frequency shift from $f=n f_{c}$ as a function of the electron-neutral collision frequency was measured and compared with the theoretical result where the electron distribution function was assumed to be $\delta$-functional.
\end{abstract}

\section{Introduction}

It was described by $\mathrm{Twiss}^{1)}$ that negative absorption of the radio wave in a plasma are expected to occur when the Cerenkov effect, the cyclotron radiation or the synchrotron radiation is a dominant process. Since then, Bekefi et al. ${ }^{2)}$ and Tanaka et al. ${ }^{3}$ discussed this phenomenon in detail and showed that the weakly ionized non-Maxwellian plasma with a large Ramsauer effect has a negative value for the absorption coefficient at the electron cyclotron frequency. Drummond et al. ${ }^{4}$ and Shimomura et al. ${ }^{5)}$ solved the Boltzmann equation with a collision term and obtained the result that the instability can occur in the vicinity of $f=0$ (for longitudinal wave) and $f=f_{c}$ (for transverse wave) at the long wavelength limit when the electron distribution is $\delta$-functional and collision frequency $\nu$ varies as $\nu \propto v^{h}$ with $h>3$.

Experimentally, one of the authors (T. I.) et al. and Tanaka et al. have shown ${ }^{6 / 7)}$ that amplification of rf wave occur for weakly ionized gases having large Ramsauer effect, in the vicinity of the electron cyclotron frequency $\left(f=f_{c}\right)$ and low frequency region $(f / \nu \leq 1)$ and it is interpreted as a negative absorption due to the stimulated cyclotron radiation and the stimulated bremsstrahlung, respectively.

In this paper, it is described that amplification of $\mathrm{rf}$ wave also occur near the electron cyclotron harmonics $\left(f=n f_{c}\right)$ in Xe and $\mathrm{Kr}$ plasmas and the frequency shift from $f=n f_{\mathrm{c}}$ is observed when the collision frequency $\nu$ becomes large. The experimental apparatus and procedure are explained in $\$ 2$, the experimental results are shown in $\$ 3$ and these

\footnotetext{
* Dept. of Applied physics
} 
results are summarized and compared with the theoretical considerations in $\$ 4$.

\section{Experimental apparatus and procedure}

Through the experiment, we used two discharge tubes; one (TUBE I) has a plane anode (20 $\mathrm{mm}$ in diameter) and an oxide-coated cathode (10 $\mathrm{mm}$ in diameter), their separation being $30 \mathrm{~mm}$, and the other (TUBE II) has an anode (15 $\mathrm{mm}$ in diameter), a cathode (10 $\mathrm{mm}$ in diameter) and meshed grid, the separations between anode and cathode, and between grid and cathode being $33 \mathrm{~mm}$ and $3 \mathrm{~mm}$, respectively. The
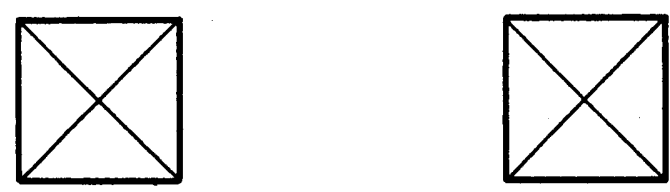

\section{Helmholtz Coil}
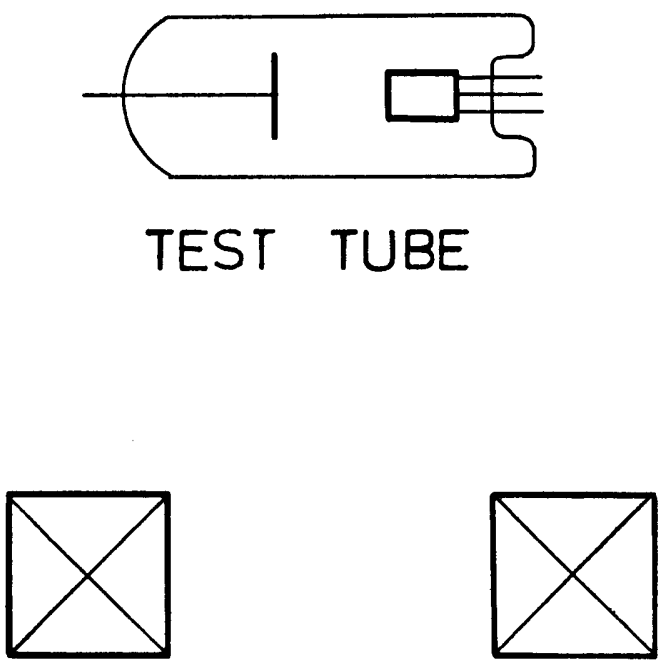

Fig. 1 (a) Schematic view of setting the test tube in the Helmholtz coil. tubes are placed in the center of the Helmholtz coil and external magnetic field is applied paralell to the tube axises. The nonuniformity of the field $A B / B$ is smaller han 3 percent through the region between the anode and the cathode, i.e., the plasma region. The plasma is produced by a $d c$ discharge in a rare gas and often an external low frequency voltage is applied to the anode in order to modurate the dc discharge. Gas pressure is varied from $10^{-1}$ Torr to several Torr. Schematic view of the apparatus described above is shown in Fig. 1.

Each tube has a movable probe and electrical oscillations in plasma are picked up from this probe and amplified and detected by a field intensity meters, whose band widths $\left(\delta f_{\text {rec }}\right)$ are about $0.5 \mathrm{MHz}$. Recieving frequency $\left(f_{\text {rec }}\right)$ is varied from $90 \mathrm{MHz}$ to $230 \mathrm{MHz}$. The output signal of the field intensity meter is traced on a XY-recorder as a function of the magnetic field intensity at fixed discharge current $I_{d}$, or is displayed on the synchroscope at fixed magnetic field and fixed discharge current. In the

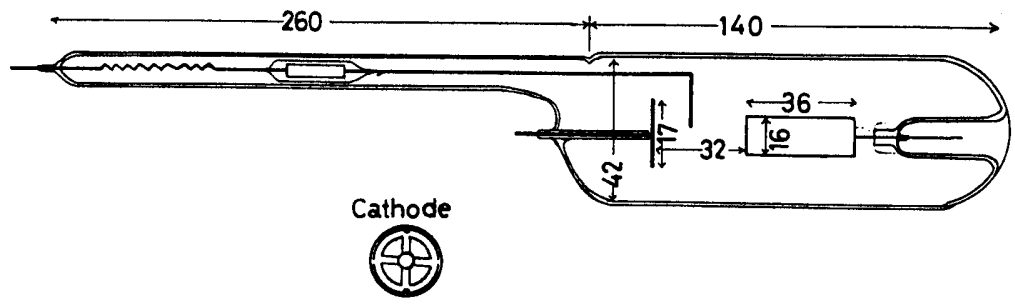

(b) Sketch of the TUBE I. 
experiment of amplification of $r f$ wave at

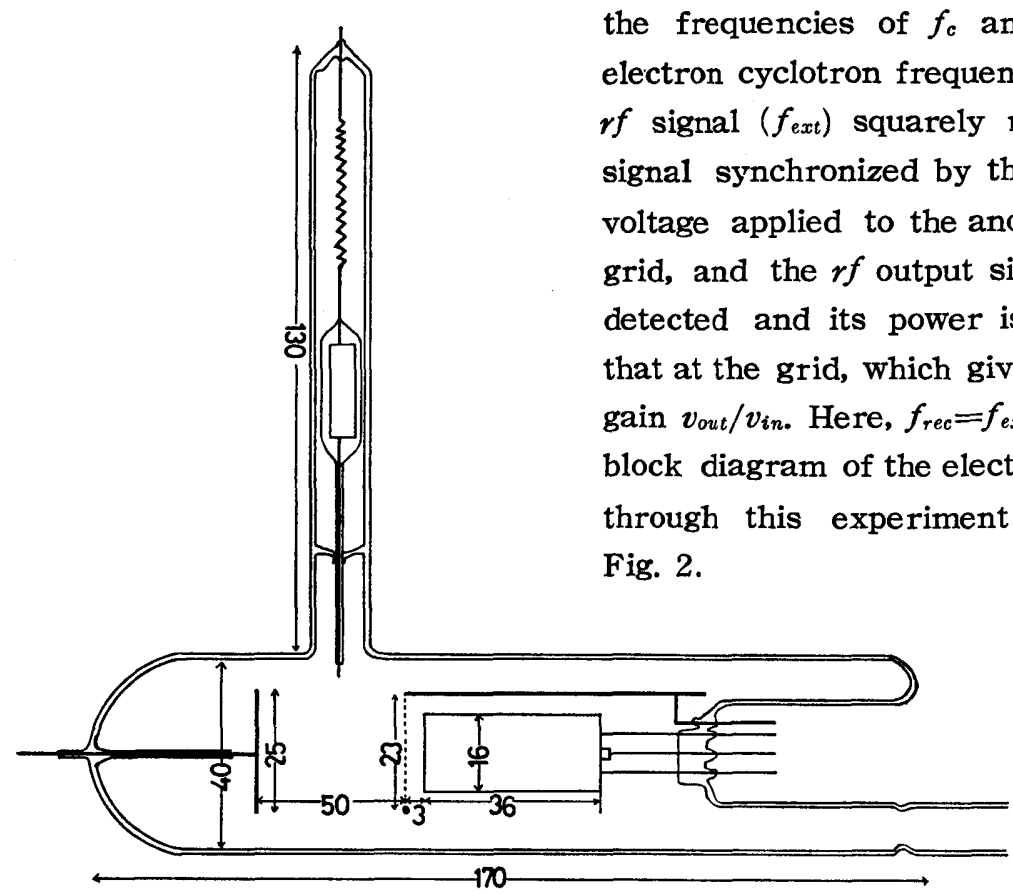

(c) Sketch of the TUBE II.

\section{Experimental results}

\subsection{Emission near the electron cyclotron harmonics}

The emisaion from the plasma is observed by means of the apparatus, whose block diagram is shown in Fig. 2(a). The emission power from the plasma picked up by the probe is traced on a XY-recorder as a function of normalized external magnetic field intensity $\left(f_{c} / f\right)$. An anomalous cyclotron emission ascribed to the negative absorption ${ }^{8)}$ is observed near the electron cyclotron frequency $\left(f=f_{c}\right)$ in $\mathrm{Xe}, \mathrm{Kr}$ and $\mathrm{Ar}$ plasma but not observed in $\mathrm{Ne}$ and $\mathrm{He}$ plasma. When a gas pressure $p$ is adjusted at the suitable value $(p=0.10$ $\sim 0.25$ Torr), the anomalous harmonic emissions are also detected near the cyclotron harmonics $\left(f=n f_{c}\right)$. In Fig. 3 , are shown a typical emission spectrum as a function of an external magnetic field intensity $\left(f_{c} / f\right)$. We have observed the anomalous harmonics up to the 4 th in Xe plasma, up to the 2nd in $\mathrm{Kr}$ plasma but only at the fundamental in

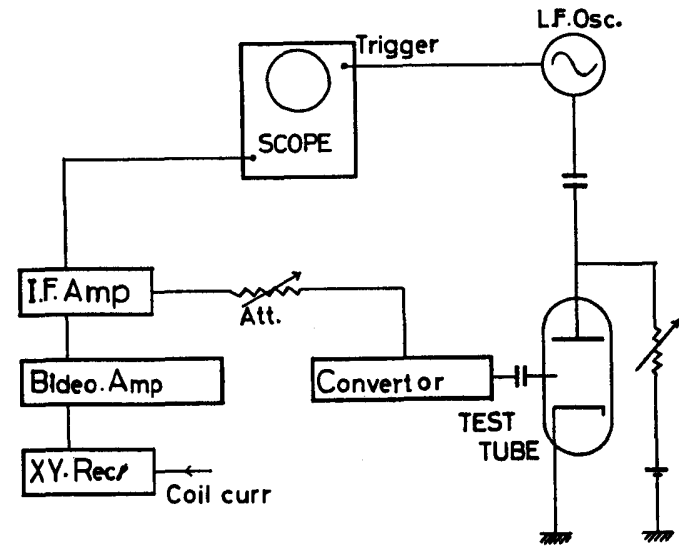

Fig. 2 (a) The block diagram of the experimental apparatus for the emission observation. 


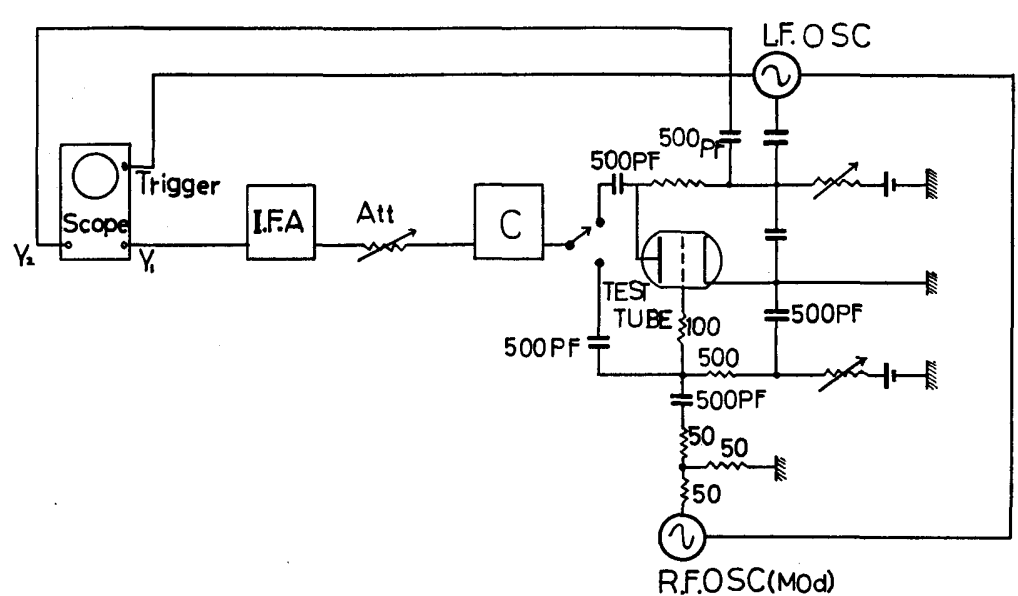

(b) The block diagram of the experimental apparatus for the observation of amplification.

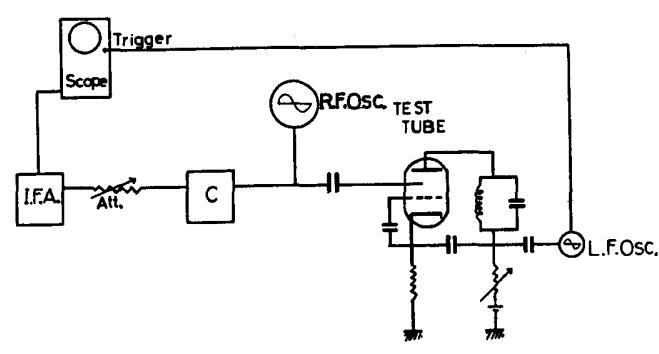

(c) The block diagram of the experimental apparatus for the observation of selfoscillation.

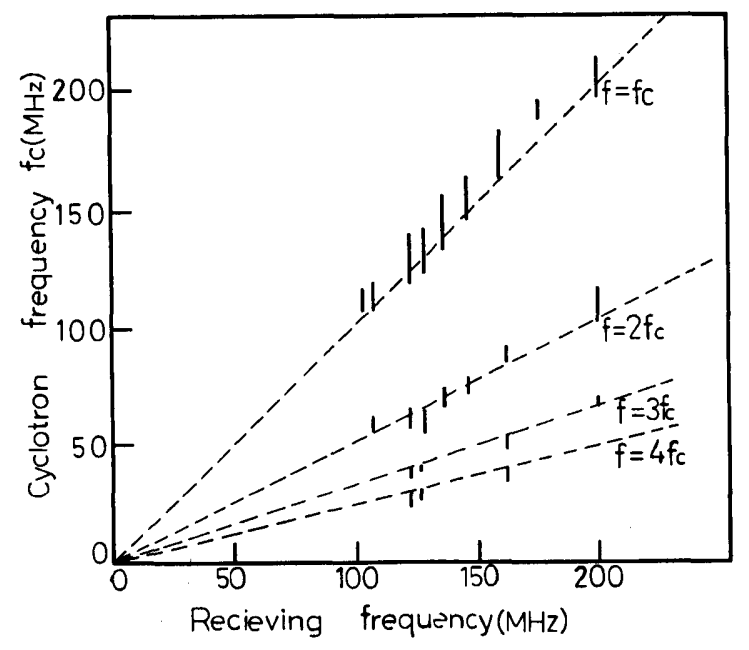

Fig. 4 The magnetic field $f_{c}$, where the emission is observed, as a function of receiving frequency.

(a) $\mathrm{Xe}, p=0.18$ Torr and $I_{d}=8 \mathrm{~mA}$,

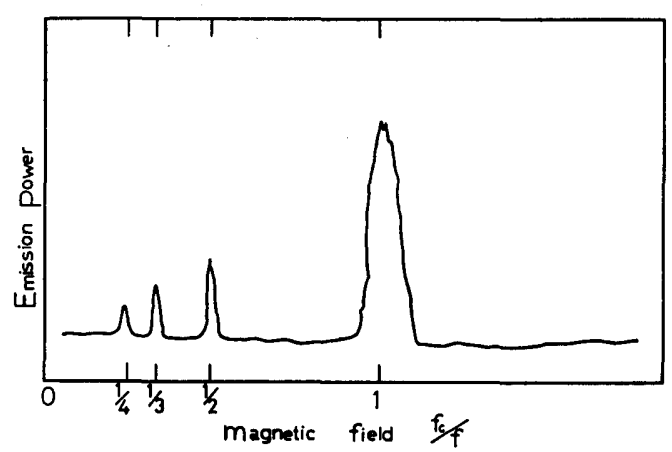

Fig. 3 Emission spectrum as a function of an external magnetic field intensity. $\mathrm{Xe}$, $p=0.18$ Torr,$f=125 \mathrm{MHz}$ and $I_{d}=8 \mathrm{~mA}$.

Ar plasma.

In Fig. 4, the magnetic field $f_{c}$, at which the anomalous harmonics are observed, is plotted as a function of the detecting frequency $f$, for the $\mathrm{Xe}$, $\mathrm{Kr}$ and Ar plasma, respectively. The characteristics of these harmonics are similar to those of the anomalous cyclotron emission described in the other paper $^{8)}$ as follows: 1) They are not stationary in time, but are pulsively emitted at a definite phase of low frequency oscillation existing spontaneously in dc discharge. 2) Their resonance lines are sharp and their widths 


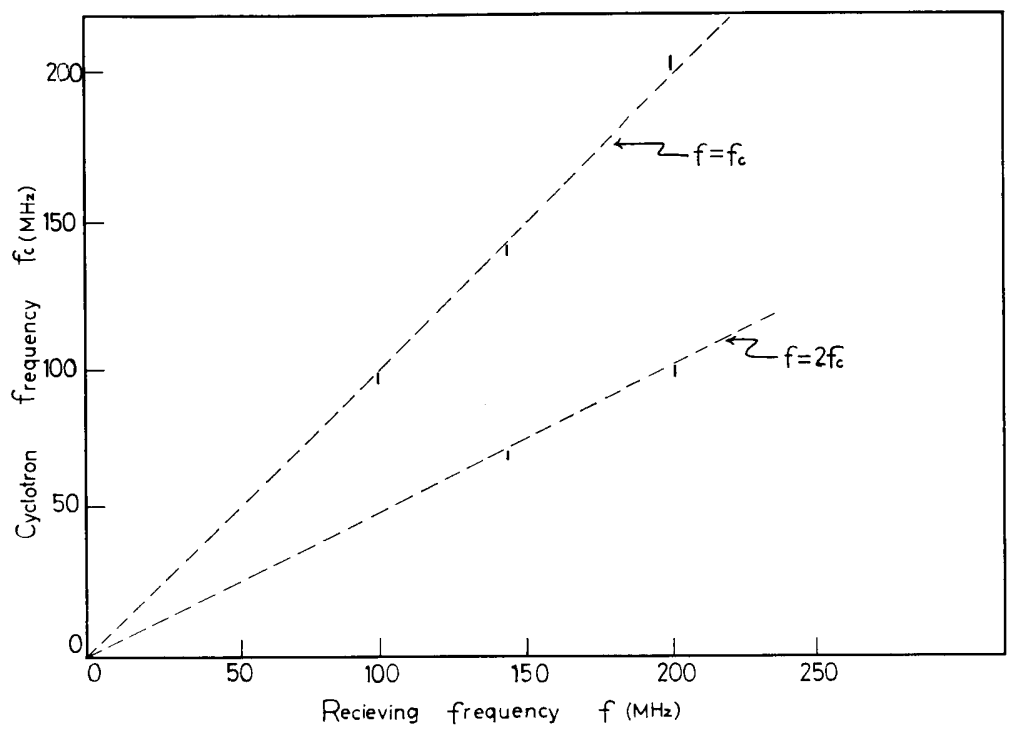

(b) $\mathrm{Kr}, p=0.20$ Torr and $I_{d}=7.5 \mathrm{~mA}$.

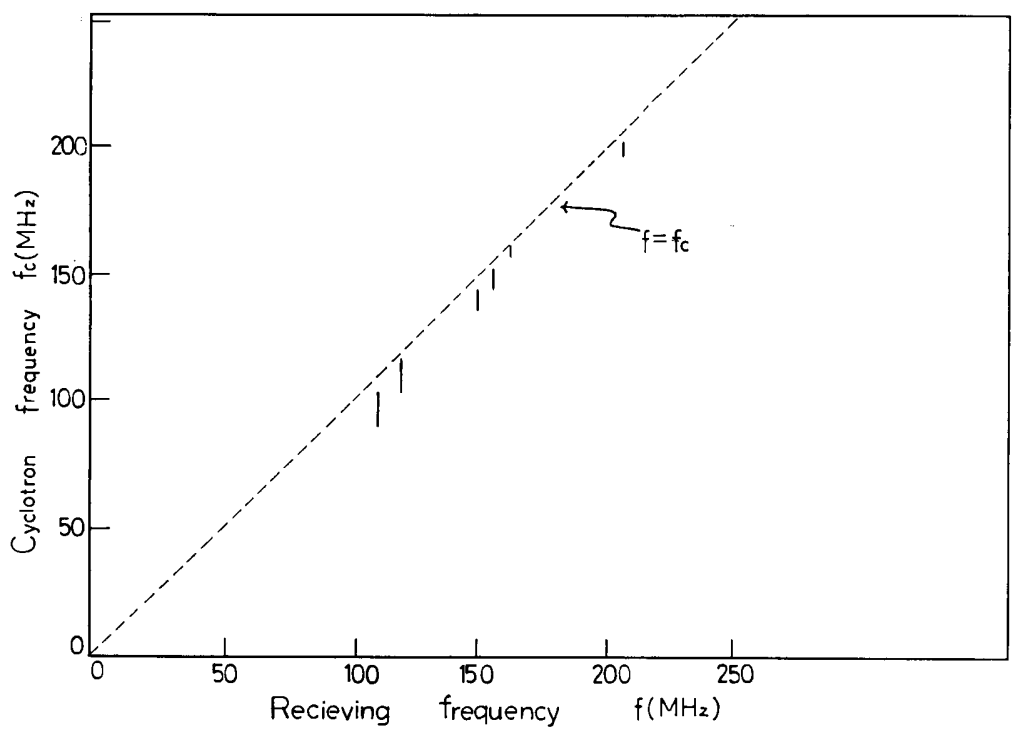

(c) Ar, $p=0.20$ Torr and $I_{d}=12 m A$.

are much narrower than those determined from the collision broadening. 3) The emission intensity is extremely larger than that of the black body, assuming that plasma electrons are in thermal equilibrium.

When an appropriate low frequency voltage is applied to the anode, the pulsed emission synchronizes with this voltage. Usually, the anomalous emissions at the fundamental $(f=93 \mathrm{MHz})$ and at the 2nd harmonic $(f=186 \mathrm{MHz})$ are both observed at the same time, as shown in Photo. 1(a). However, if the applied low frequency voltage appropriately modulates the $d c$ discharge, they do not occur at the same time (Photo. 1(b)). 


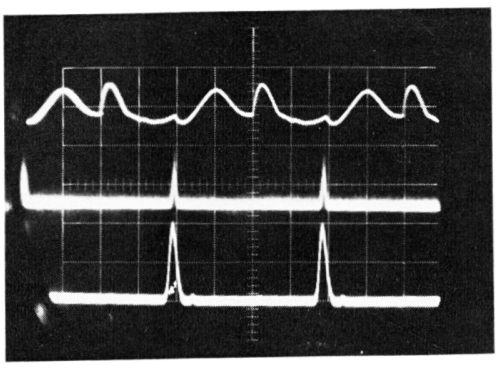

(a)

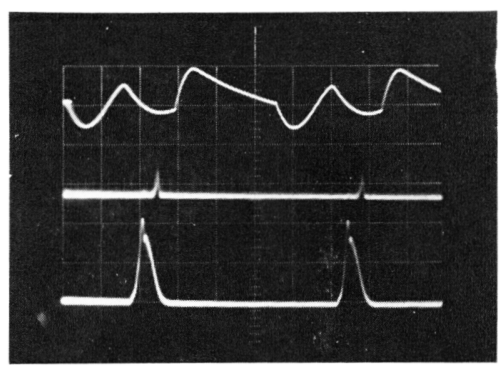

(b)

Photo. 1 The anomalous emissions. Upper trace: low frequency signal at the anode. Middle trace: emission power at the cyclotron frequency. $f=f_{c}=93 \mathrm{MHz}$. Lower trace: emission power at the second harmonic. $f=2 f_{c}=186 \mathrm{MHz}, 200 \mu \mathrm{sec} / \mathrm{div}$.

(a) Xe, $p=0.21$ Torr and $I_{d}=7.5 \mathrm{~mA}$.

(b) Xe, $p=0.18$ Torr and $I_{d}=7.0 \mathrm{~mA}$.

\subsection{Amplification of the radio wave near the 2nd harmonic}

The amplification of $r f$ wave is observed by using the apparatus as shown in Fig. 2 (b). An appropriate external low frequency voltage is applied to the anode in order to modulate the dc discharge and such a plasma that amplifies $r f$ wave is produced. Measurements are carried out by a field intensity meter at a fixed frequency of $f=93$ $M H z$ or $186 \mathrm{MHz}$. The external $r f \operatorname{signal}\left(f_{e x t}\right)$ squarly modulated by $l f$ signal, is fed to the grid and the $r f$ output signal at the anode is detected. The lower trace in Photo. 2 (a) and (b) show respectively the input signal at grid and the output signal at anode. Here, the cyclotron frequency of the external magnetic field is set at $\mathrm{f}_{c}=93 \mathrm{MHz}$ and the frequency of $r f$ signal is adjusted te be twice $\mathrm{f}_{c}$, i.e., $f=f_{e x t}=2 f_{c}=186 \mathrm{MHz}$.

The upper traces show the anode potential modulated by if voltage. Comparing the output signal $\left(v_{\text {out }}\right)$ with the input $\operatorname{signal}\left(v_{\text {in }}\right)$, it is known that the input signal is amplified at a certain phase of $l f$ voltage $\varphi_{1}$. The $r f$ voltage gain is about $4 d B$. Since the ratio of the input impedance to the output one is estimated to be $3 d B$ from Fig. 2 (b), the

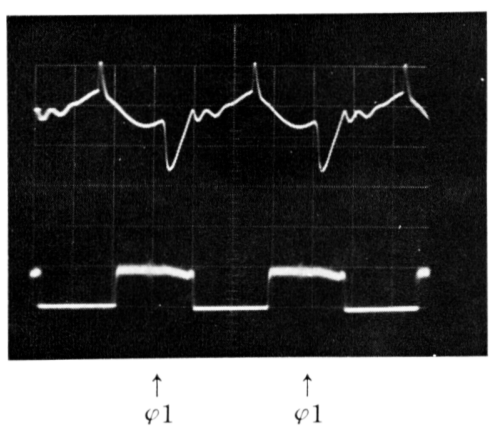

(a)

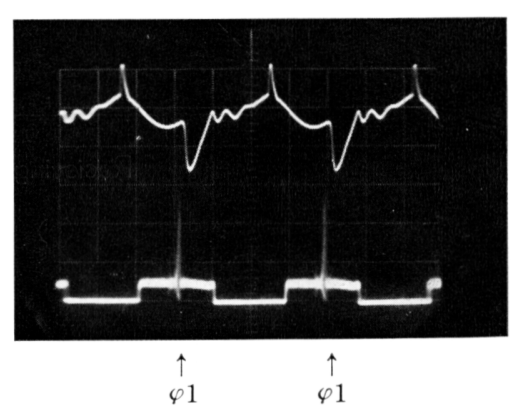

(b)

Photo. 2 (a) Upper trace: low frequency signal at the anode. Lower trace: $r f$ input signal at the grid.

(b) Upper trace: low frequency signal at the anode.

Lower trace: $r f$ output signal at the anode.

Xe, $p=0.47$ Torr $, f=f_{\text {ext }}=186 \mathrm{MHz}, f_{c}=93 \mathrm{MHz}, I_{d}=3.6 \mathrm{~mA}$ and sweep: $200 \mu \mathrm{sec} / \mathrm{div}$. 
power gain is about $7 d B$. Measurements at the receiving frequency of 93 $\mathrm{MHz}$ show that the amplification occurs also at the fundamental frequency $(f=$ $f_{\text {ext }}=f_{c}=93 \mathrm{MHz}$ ), by about $9 \mathrm{~dB}$.

Fig. 5 shows the voltage gain as a function of $r f$ input power both at the cyclotron frequency and its 2 nd harmonic. Usually, the input power, where the amplification occurs at the 2nd harmonic, is smaller than that at the fundamental. Exceeding a certain value of input power, the voltage gain decreases with increasing input power, and a saturation effect is expected as seen in Fig. 5.

\subsection{Observation of the self-oscilla-} tion near $f=n f_{c}$

As shown in Fig. 2 (c), the $r f$ signal from the anode is fed back to the grid in order to make the self-oscillation at the resonant frequency. Near the fun-

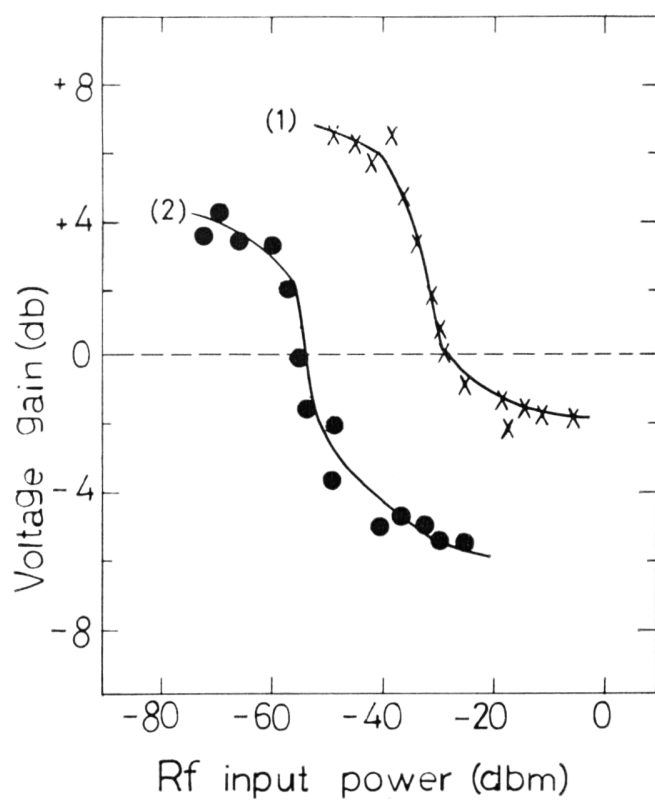

Fig. 5 The voltage gain as a function of $r f$ input power at the cyclotron frequency and its second harmonic.

(1) for the cyclotron frequency, $f_{r e c}=f_{e x t}$ $=f_{c}=93 \mathrm{MHz}$.

(2) for the second harmonic, $f_{r e c}=f_{\text {ext }}=2 f_{c}$ $=186 \mathrm{MHz}$. damental frequency $\left(f=f_{c}\right)$ and the 2 nd harmonic $\left(f=2 f_{c}\right)$, when the value of capacity of the external circuit is adjusted at the appropriate value, the $r f$ oscillation occurs strongly. In Photo. 3 (a), is shown the $r f$ signal from the probe at the fundamental frequency. In Photo. 4, are shown the direct observation of $r f$ signal in the case of particularly large one, by means of $100 \mathrm{MHz}$ synchroscope, which shows that the oscillation is sinusoidal and its peak-to-peak-voltage is about $0.04 \mathrm{mV}$. When the external signal whose frequency is near the recieving frequency $\left(f_{\ell x t} \approx\right)$, the beat of the both signals,

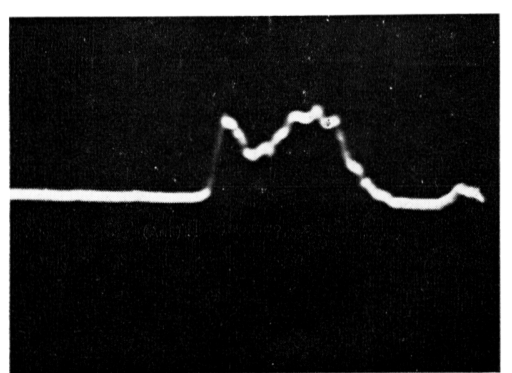

(a)

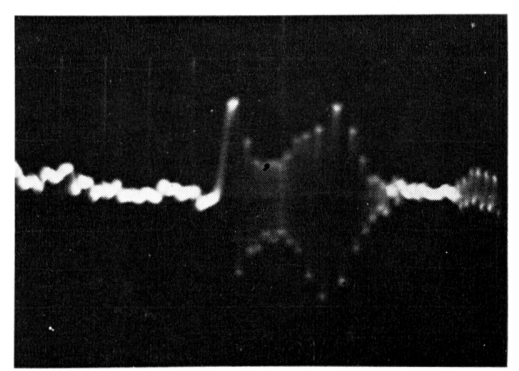

(b)

Photo. 3 (a) Anomalous emission pulse.

(b) Beat of an anomalous emission with an external signal.

Xe, $p=0.27$ Torr $, I_{d}=4.2 \mathrm{~mA}, f=f_{c}=93 \mathrm{MHz}$ and sweep: $100 \mu \mathrm{sec} / \mathrm{div}$. 
i.e., the external signal and the oscillation signal in a plasma-external-circuit system, are observed as shown in Photo. 3 (b). From this photograph, it is confirmed that the oscillation is coherent but the frequency is varied in a single pulse. The variation of frequency $\delta f$ seen in this photograph is about $140 \mathrm{KHz}$ and comparable with the band width $\delta f_{\text {rec }}$ of a field intensity meter used here. This value of $\delta f$ is often larger than that of $\delta f_{\text {rec }}$ and reach several $\mathrm{MHz}$.

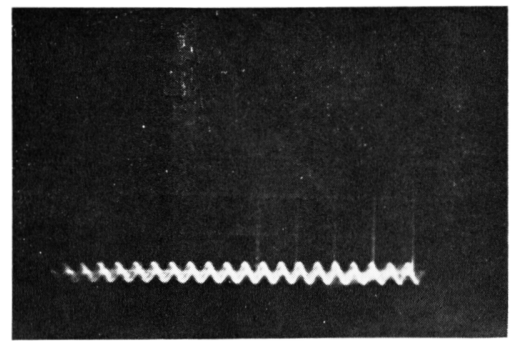

Photo. 4 Direct observation of the oscillation due to the negative absorption at the cyclotron frequency. sweep : $0.05 \mu \mathrm{sec} / \mathrm{div}$.

\subsection{Frequency shift from $f=n f_{c}$ as a function of background pressure $p$}

Fig. 6 shows the emission spectrum as a function of the magnetic field intensity $\left(f_{c} / f\right)$ with $I_{d}$ as a parameter. It is seen from this figure that the value of the magnetic field intensity where the emission is observed is shifted with increasing $I_{d}$. This frequency shift $\left(\Delta f_{c} / f\right)$ can not be interpreted as the nonuniformity of the external magnetic field $(\Delta B / B)$ through the plasma region, because $\Delta f_{c} / f$ is about ten percent and much larger than $\Delta B / B\left(\sim 3\right.$ percent). The magnetic field intensity $f_{c} / f$ where the emission is observed is plotted as a function of $I_{d}$ in Fig. 7. From this figure, the maximum value of this shift $\left(\Delta f_{c} / f\right)_{\max }$ are determined for the certain value of back ground pressure $p$. By measuring for the various values of $p$, it is knorn that $\left(\Delta f_{c} / f\right)_{\max }$ varies as a function of $p$, as shown in Fig. 8.

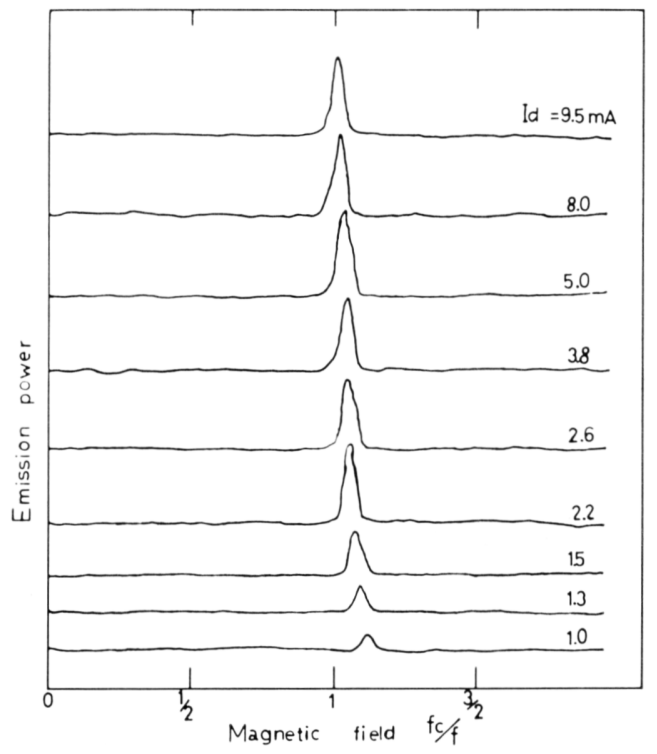

Fig. 6 Emission spectrum as a function of the magnetic field intensity with $I_{d}$ as a parameter. Xe, $p=0.75$ Torr and $f=150 \mathrm{MHz}$.

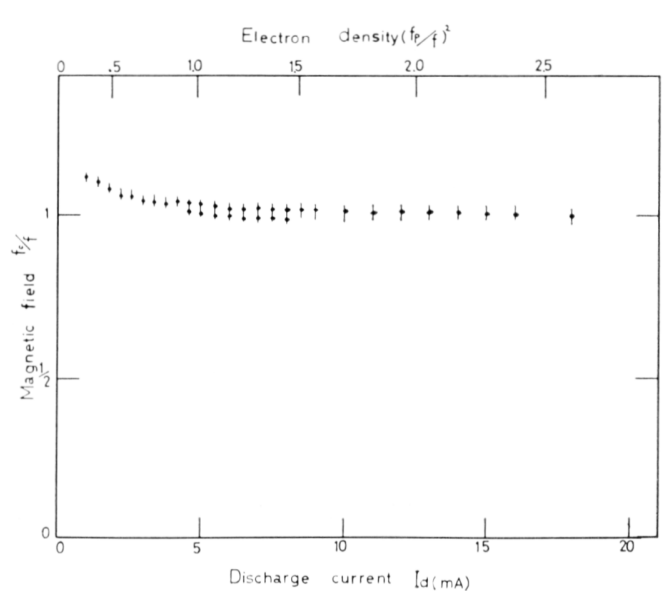

Fig. 7 The magnetic field intensity $f_{c} / f$, where the emission is observed, as a function of $I_{d}$. Xe, $p=0.75$ Torr and $f=150$ $\mathrm{MHz}$. 


\section{Discussion}

The experimental results described in $\$ 3$ are summarized as follows :

I) Negative absorption phenomenon due to the Ramsauer effect occurs not only near the cyclotron frequency but also near its 2 nd harmonic. The anomalous emission ascribed to this phenomenon is observed at the higher harmonics up to 4 th.

II) The system composed from the plasma and external circuit selfoscillates pulsively at the cyclotron frequency and its 2nd harmonic, but the frequency shift $(\delta f \sim$ several $M H z)$ is observed in a single pulse.

III) The frequency at which the anomalous emission is observed shifts with increasing $I_{d}$ and the maximum value of this shift $(\Delta f / f)_{\max }$ increases with a background pressure $p$.

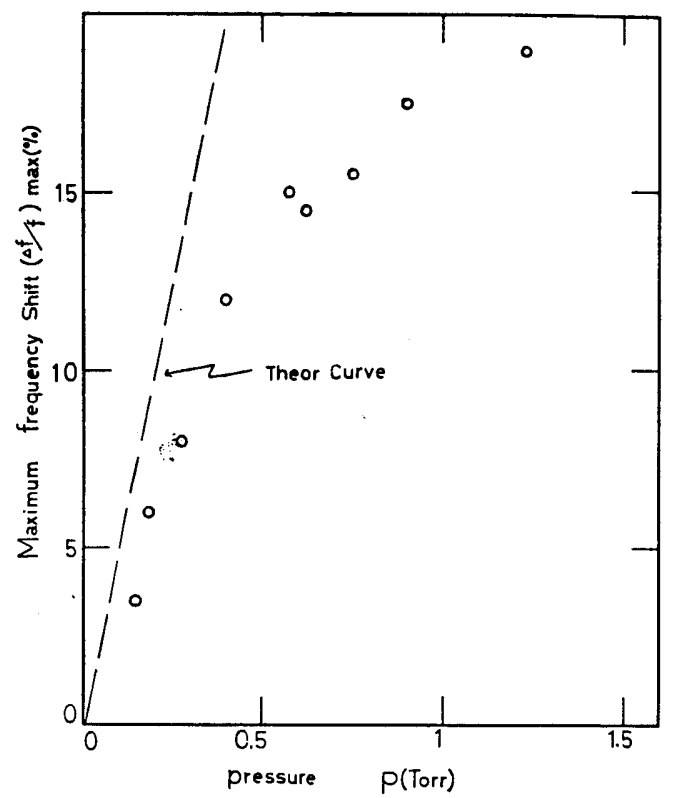

Fig. 8 Maximum frequency shift as a function of pressure. Theoretical curve is drawn by using Eq. (5) and substituting the experimental conditions to it.

On the other hand, the possibility of the negative absorption near the cyclotron harmonics is discussed theoretically elsewhere ${ }^{9}$. Following them, the assumptions are as follows :

1) The zeroth order distribution function is $\delta$-functional;

$$
f_{o}=\frac{1}{4 \pi v^{2} o} \delta\left(v-v_{o}\right) \text {. }
$$

2) The collision frequency of an electron with neutral atoms is proportional to $v^{h}$;

$$
\nu \propto v^{h} .
$$

3) The collision term of the Boltzmann equation is assumed as follows;

$$
\left(\frac{\partial f}{\partial t}\right)_{c o l l}=-\nu(v) f(r, v, t)+\frac{1}{4 \pi} \int \nu(v) f(r, v, t) d \Omega,
$$

where $d \Omega$ is the elementary solid angle in velocity space.

By solving the Boltzmann equation under these assumptions, the dispersion relation for the extraordinary wave near the cyclotron harmonics $\left(f \cong n f_{c}\right)$ with $k^{2} c^{2} / \omega^{2} \gg 1$ is as follows ;

$$
\omega\left(\omega-n \omega_{c}+i \nu\right)^{2}=\frac{n^{2}}{(2 n) !} \omega_{p^{2}}^{2} \lambda_{0}^{2 n-2}\left(\omega-n \omega_{c}+i \frac{2 n+1-h}{2 n+1} \nu_{0}\right),
$$

where $\omega=2 \pi f, k$ is a propagation constant, $\lambda_{0}=k v_{0} / \omega_{c}$ and $\nu_{0}=\nu\left(v_{0}\right)$. It is confirmed from this equation, that the negative absorption occur near the nth cyclotron harmonics, for the extraordinary wave if $h$ is larger than $2 n+1$. Under the same assumptions as mentioned above, the negative absorption for the ordinary wave with $k^{2} c^{2} / \omega^{2} \gg 1$ is exp- 
ected to occur near the nth harmonics if $h$ is larger than $2 n+3$.

We will compare this theoretical consideration with the experimental results in order to give them the qualitative interpretation, though it is combined with the severe assumptions.

1) The wavelength is considered to be comparable with the plasma scale and the condition of $k^{2} c^{2} / \omega^{2} \gg 1$ is satisfied for our plasma.

2) The observed wave do not propagate perpendicularly to the magnetic field. However, as $k$ has a component perpendicular to the magnetic field, it is expected that the theory mentioned above is fitted to our experiment qualitatively.

3) The assumption of $\nu \propto v^{h}$ describes the Ramsauer effect expected in our plasma.

4) The assumption that the distribution function is a $\delta$-functional, describes the population inversion expected in our plasma, qualitatively.

5) In Xe plasma whose value of $h$ is considered to be largest among rare gases,

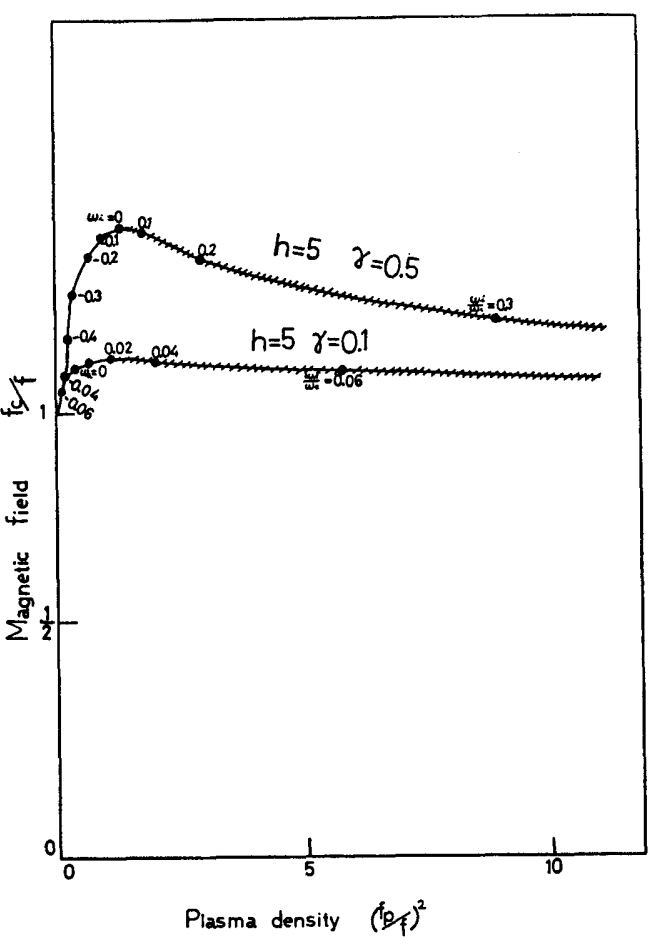

Fig. 9 Dispersion curves with $r\left(=\nu_{0} / \omega_{r}\right)$ as a parameter. Imaginary part of the frequency $\left(\omega_{i} / \omega_{c}\right)$ is shown on these curves. In the hatched region, where $\omega_{i} / \omega_{c}$ is positive, the negative absorption is expected to occur. the highest order harmonics is observed. This is consistent with the theoretical results.

6) In Fig. 9, are plotted the dispersion curve from Eq. (4). This curve shows that the magnetic field where the negative absorption occurs, shifts with the plasma density. This interpretes the experimental results shown in Fig. 7, qualitatively.

7) From Eq. (4), the maximum frequency shift $(\Delta f / f)_{\max }$ is calculated as follows ;

$$
(\Delta f / f)_{\max } \approx 0.30 \frac{h}{2 n+1} \frac{\nu}{f}
$$

This curve is plotted in Fig. 8 and its tendency is consistent with the experimental one.

From the reasons mentioned above, the theory interpretes the experimental results qualitatively. After all, we want to emphasize through the paper that the negative absorption occur not only near the electron cyclotron frequency but also near the cyclotron harmonics.

\section{Acknowledgement}

The authors wish to express their sincere thanks to Professor S. Tanaka and Dr. Y. Terumichi of Kyoto University for their valuable discussions. 


\section{References}

1) R. Q. Twiss : Australian J. Phys. 11 (1956) 564.

2) G. Bekefi, J. L. Hirshfield and S. C. Brown : Phys. Fluids 4 (1961) 173.

3) S. Tanaka and K. Mitani : J. Phys. Soc. Japan 19 (1964) 1376.

4) J. E. Drummond, D. J. Nelson and J. L. Hirshfield : to be published.

5) N. Shimomura, K. Mitani and S. Tanaka : J. Phys. Soc. Japan 21 (1966) 1372.

6) Y. Terumichi, T. Idehara, I. Takahashi, H. Kubo and K. Mitani : J. Phys. Soc. Japan 20 (1965) 1705 .

7) S. Tanaka and K. Takayama : J. Phys. Soc. Japan 22 (1967) 310.

8) For example, this is reported by S. Tanaka et al. in J. Phys. Soc. Japan 18 (1963) 1810.

9) T. Idehara and R. Sugaya : J. Phys. Soc. Japan 23 (1967) 1122. 